\title{
THE RETICULAR PERIVASCULAR TISSUE OF THE CENTRAL NERVOUS SYSTEM
}

\author{
BY \\ J. W. MILLEN and D. H. M. WOOLLAM \\ From the Department of Anatomy, University of Cambridge
}

Since changes occurring in the nerve cell body and its processes in consequence of trauma or disease produce effects which must obviously be of fundamental importance to the functioning of the nervous system as a whole, the central position in the field of neuropathological research has naturally for many years been occupied by the neuron. The focusing of attention on the neuron has had, however, the unfortunate effect of producing a consequential disregard of other elements. The neglect to which the neuroglial elements of the nervous system were for a long time subject was remedied, at least to some extent, when adequate staining methods were developed for the demonstration of these structures. The evolution of gold and silver impregnation methods enabled great advances to be made in the understanding of the role played by the neuroglia in normal and pathological processes. At the present time, although it is well recognized that perivascular accumulation of cells are a feature of such diseases as encephalitis and poliomyelitis, the relationship of such exudates to the blood vessel and its sheath on the one hand, and to the nervous tissue proper on the other, has been subject to considerable confusion, and umuch msinterpretation. It is the purpose of this paper to suggest that the application of a suitable staining method can lead to the better understanding of the extent and intimate associations of the reticular perivascular sheath, its relationships to the meninges, to the true and artefact perivascular spaces, and thus of its reaction to and implication in disease processes.

The first investigators to recognize that the blood vessels entering the central nervous system were accompanied by reticular perivascular sheaths derived from the lepto-meninges were Key and Retzius (1876) who described the sheaths as formed by visceral and parietal layers continuous with the linings of the subarachnoid space. They injected gelatine coloured with Berlin blue into the subarachnoid space of human cadavers and, as a result of their experiments, were in no doubt that the space between the two layers of this reticular leptomeningeal sheath was freely in communication with the general subarachnoid space. Earlier workers (Virchow, 1851 ; Robin, 1859 ; His, 1865), and indeed some later ones also (Bevan Lewis, 1889 ; Bruce and Dawson, 1911) appear to have been preoccupied with the search for the lymphatics of the brain. Most of these workers failed to appreciate that the tissue surrounding the blood vessels was lepto-meningeal in origin and regarded this sheath simply as the tunica adventitia of the vessel. They believed that the perivascular spaces were lymphatics related to the tunica adventitia of the blood vessels entering the central nervous system. There was, however, no general agreement as to the exact situation of these spaces. Even Virchow (1851) and Robin (1859), whose names are conjoined in the eponymous nomenclature of the perivascular space, did not agree in their descriptions of its situation : - Virchow regarded the spaces as "sub-adventitial" and open to the subarachnoid space, while Robin believed them to be closed and "intra-adventitial" spaces. Cushing (1926) indeed remarked in his Studies in Intracranial Physiology and Surgery that " these distinguished investigators originally gave such casual descriptions of the spaces in question that it is surprising that they should have become eponymic." Tuke (1894) recognized the meningeal origin of the perivascular sheaths but regarded the perivascular space as lymphatic in nature and as responsible for the removal of waste and superfluous material ; indeed he went so far as to suggest that the perivascular space was the beginning of a lymph path which ultimately ended in the lymph glands of the head and neck.

Most of these workers based their descriptions of the perivascular sheaths upon the examination of pathological material and were handicapped by the inadequate histological techniques available to them. Furthermore, with the notable exception of His 
(1865), they seldom attempted to supplement their descriptions by the employment of experimental procedures involving the use of indicator substances. His used a multiple intracerebral puncture technique and was able to inject spaces around the blood vessels within the nervous system. He recognized that these spaces were not the true perivascular, or Virchow-Robin, spaces, and, since in his opinion they were bounded by cells of an endothelial type and communicated with subarachnoid "lakes" situated between the brain tissue and the surface pia mater (epispinal spaces of His), he termed them "lymphatics". Unfortunately the clear distinction drawn by His between the spaces he injected and the Virchow-Robin spaces was soon lost sight of and subsequent workers appear to have regarded them as identical. Thus, far from clarifying the picture of the reticular sheath and perivascular space around the blood vessels, the researches of His increased rather than diminished the confusion surrounding their description.

The absence of agreement as to the anatomy of the lepto-meninges renders more difficult a clear understanding of the nature, identity, and connexions of the reticular perivascular sheath. By some authorities stress is laid on the separate identity of the pia mater and the arachnoid mater and, in consequence, the exact identification of the layers forming the perivascular sheath becomes a question of some importance. Tuke (1894) regarded the vessels as carrying prolongations of the pia mater with them into the brain substance. He thought that this pial sheath replaced the adventitial layer of the blood vessels within the central nervous system, and that it surrounded the blood vascular system to its ultimate branches. Mott (1910) held views on the nature of the perivascular sheath very similar to those of Tuke. He was the first to consider that there were differences in the nature and extent of the sheaths between the arteries and veins. Mott thought that the perivascular sheaths were better marked around the arteries than around the veins : he also noted that the sheath was continued over the capillaries. Hughson (1925) believed that the outer wall of the perivascular sheath was formed by the turning inwards of the pia mater, whilst the layer on the vessel itself, forming the inner layer of the sheath, was derived from the cells of the arachnoid mater. Schaltenbrand and Bailey (1928) in their descriptions referred to the "pia-arachnoid", apparently regarding the lepto-meninges as forming a single entity. They did not concern themselves greatly with the subdivision of the perivascular sheath into pial and arachnoid components and were content to regard the sheath as formed by a sheet of reticular connective tissue equivalent to the piaarachnoid. At the same time the pial nature of the outer layer of the sheath was implicit in their description of this layer as a "piaglialmembran". One of the most recent accounts of the reticular perivascular sheaths was that given by Patek (1944). He reaffirmed the description of Key and Retzius (1876) and regarded the arachnoid mater as forming the inner layer of the perivascular sheath, but differed from them in regarding the pia mater as providing only the peripheral part of the outer layer of the sheath. In his view the deeper parts of the outer layer had the form of a fenestrated membrane : this standpoint will be considered later.

A consideration of the findings outlined above suggested that an experimental technique which combined the use of an indicator substance with a staining method specific for reticular tissue appeared likely to throw new light on the nature and extent of the reticular perivascular sheath, and its relationship to the perivascular spaces.

\section{Material and Methods}

Histological examinations were carried out on 40 albino rats and on human material from the central nervous systems of a full-term foetus and of patients who had died following epidemic encephalitis and anterior poliomyelitis.

Newborn albino rats were used for experimental observations employing an indicator substance introduced into the cranial subarachnoid space. Briefly this method consisted in the daily injection, from birth and continuing over a period of about three weeks, of a small amount $(0.05$ to $\mathbf{0 . 1} \mathrm{ml}$.) of colloidal carbon into the cranial subarachnoid space. The injection caused only a very temporary disturbance from which the animal recovered in a few minutes. The alteration in intracranial pressure produced by the quantity of the indicator employed was evidently quite compatible with life, and might therefore be regarded as within the limits of normal physiological pressures. At the end of three weeks the animals were sacrificed. The brains and spinal cords were removed carefully, keeping the lepto-meninges intact as far as possible, and subjected to the same histological examination as the human material.

The tissues were fixed, embedded in paraffin, and sectioned in the usual manner. A variety of stains was employed, including haematoxylin and eosin, toluidine blue, Mallory's trichrome stain, and a modification of Bielschowsky's stain. It was found that the application to the central nervous system of a modification of the stain for reticular and collagenous fibrils described by Long (1948) yielded the most valuable information. This method gave excellent results with formalin-fixed material.

The details of the method are as follows. The sections are dewaxed and carried through descending grades of alcohol to distilled water. Then they are treated with 
$0.25 \%$ aqueous solution of potassium permanganate for five minutes, washed in running water for five minutes, and rinsed in distilled water. They are placed in $5 \%$ oxalic acid for five minutes and then washed in running water for five minutes. They are washed in three changes of distilled water for 10 minutes. The sections are placed in a $5 \%$ solution of silver nitrate for 12 hours in the dark, washed in four changes of distilled water, and treated with ammoniacal silver carbonate in an oven at 40 to $45^{\circ} \mathrm{C}$. for 30 minutes. The ammoniacal silver carbonate is prepared as described by Long but diluted to a $25 \%$ solution by the addition of double glass-distilled water. When the sections are removed from this solution they should be golden brown. Each slide is dipped separately in a jar containing four drops of ammonia in $500 \mathrm{ml}$. distilled water and then transferred to $1 \%$ neutral formalin for 10 minutes. The sections are washed in running water for 10 minutes and then rinsed in distilled water. The sections are toned in $0.005 \%$ gold chloride for 15 minutes, washed in distilled water, and fixed for five minutes in $5 \%$ hypo., and washed in running water. The sections are stained with $1 \%$ neutral red for 10 minutes at room temperature and differentiated in $70 \%$ alcohol. They are dehydrated, cleared, and mounted in neutral balsam. The reticular perivascular sheath and lepto-meninges are stained a purplish-black, the neurons red, and the background pink.

The use of this modification of Long's stain makes it possible to stain quite specifically the reticular perivascular tissue and to identify with considerable certainty its relationship to other structures.

\section{Results}

Normal Material.-The description which follows of the arrangement of the reticular tissue related to the blood vessels of the central nervous system is derived equally from the examination of the nervous system of the experimental rats and from the human full-term foetal material except where indicated. There do not appear to be any significant differences between man and the rat in regard to this reticular tissue, nor would one expect to find such differences. The account is a general one based upon the examination of a large number of sections from many animals and individual specimens will not be described.

Each blood vessel carries with it into the central nervous system a reticular sheath which is continuous with the lepto-meninges and is clearly derived from them (Fig. 1). Except over the larger segmental branches this reticular tissue is small in amount and appears as a thin layer immediately external to the muscle cells of the tunica media. It may be noted here that, within the central nervous system, no distinct adventitial layer can unequivocally be recognized in the walls of the blood vessels. The reticular sheath extends over the arteries, the arterioles, the larger capillaries, the venules, and

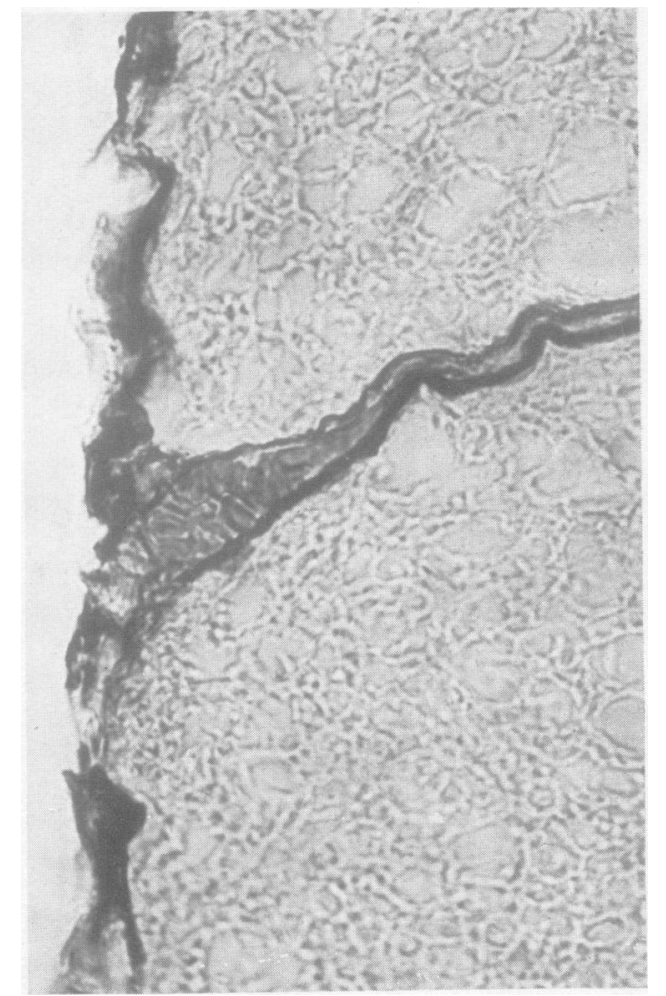

FIG. 1.-Transverse section through the spinal cord of a young rat. The section shows a blood vessel entering the cord, and demonstrates the continuity of the pia-arachnoid with the reticular perivascular sheath. Stained by a modification of Long's stain. $\times 760$.

the segmental veins. In addition, it is not possible to be certain whether a very thin layer may not also separate even the smaller capillaries from the nervous tissue proper.

In normal material the two-layered nature of the reticular perivascular sheath is only apparent in relation to the larger segmental vessels. The smaller vessels present no evident separation of the reticular sheath into two layers, although the examination of pathological material suggests that such a separation can be made to appear by the splitting of the reticular sheath.

In many specimens, particularly when intravenous hypertonic saline has been administered to the animal before death, the blood vessels are separated from the nervous tissue itself by spaces of divers dimensions. The outer walls of these spaces show no lining membrane, but their inner walls are formed by the external surface of the reticular sheath, and are separated from the vessels by the whole thickness 


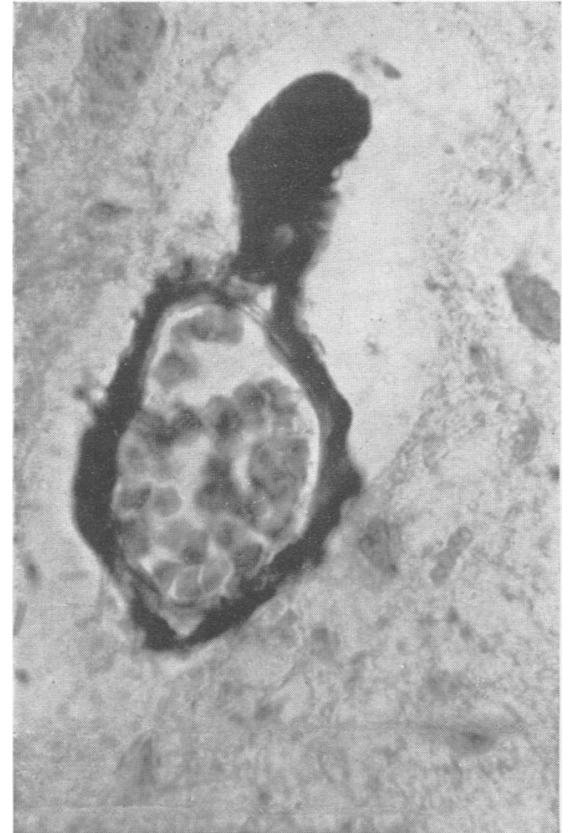

FIG. 2.-Transverse section through the spinal cord of a full-term human foetus. The reticular sheath of the blood vessel is separated from the nervous tissue by an empty trabeculated space, the artefact perivascular space. Stained by a modification of Long's stain. $\times 800$.

of the perivascular tissue (Fig. 2). These spaces can often be followed to the surface of the spinal cord where their continuity with the epispinal space of $\mathrm{His}$ is unequivocal. There can be no doubt that these spaces are in fact the artefact perivascular spaces and not the true perivascular spaces of Virchow-Robin.

The subarachnoid injection during life of an indicator such as colloidal carbon makes possible the identification of the perivascular spaces in histological preparations by the observation of small particles of carbon lodged alongside the blood vessels in the depths of the nervous tissue. In favourable sections, when the lepto-meninges have been carefully preserved, it is possible to demonstrate with certainty the continuity of this carbon with that lying in the general subarachnoid space (Fig. 3). These perivascular carbon particles are lying between the two layers of the reticular perivascular sheath and are separated from the artefact perivascular space by the outer layer of this reticular tissue. It follows that the true perivascular space of Virchow-Robin, which opens freely exteriorly into the subarachnoid space, lies between the two layers of the reticular perivascular sheath.
One of the most striking conclusions that is compelled from the examination of sections of the central nervous system of rats which have received colloidal carbon during life is the relative paucity of the perivascular spaces. Generally only the larger vessels entering or leaving the nervous tissue show perivascular carbon particles. These vessels are few, sometimes only one or two in $0.1 \mathrm{~mm}$. of the spinal cord. It follows that, although potential spaces may exist, only a small number of true perivascular spaces can, in fact, be observed in normal nervous tissue.

Pathological Material.-The pathological material examined comprised sections from the brains and spinal cords of patients who had died following attacks of anterior poliomyelitis or epidemic encephalitis. Since only the part played by the reticular perivascular sheaths in the disease processes was of interest in this investigation no particular

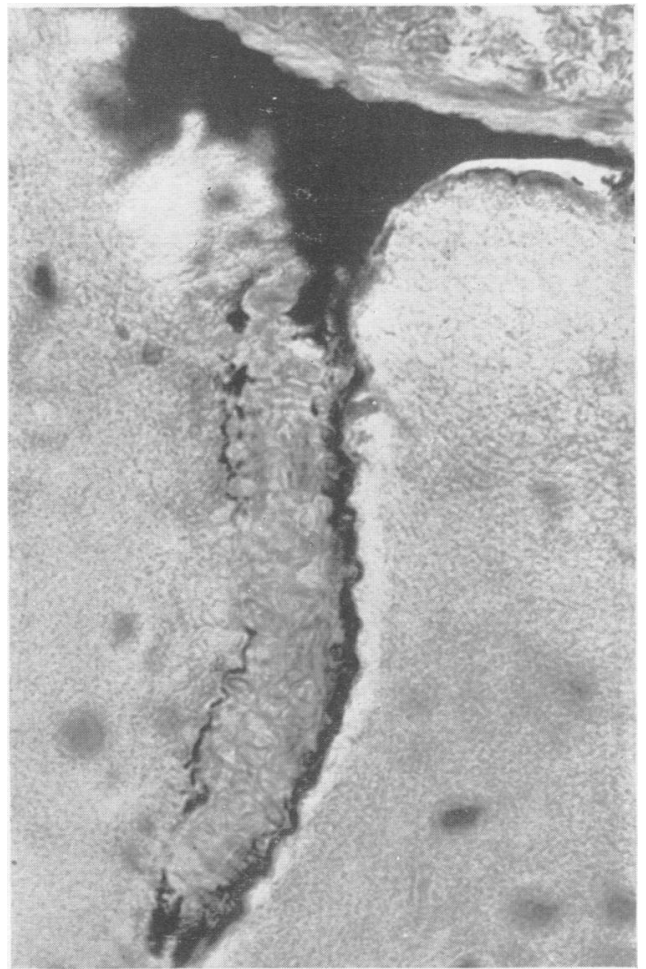

FIG. 3.-Section through the cerebral hemisphere of a young rat which had received a daily subarachnoid injection of colloidal carbon from birth until the age of 21 days when it was killed. The continuity of the carbon in the true perivascular space with that in the subarachnoid space is shown. The clear space separating the colloidal carbon from the brain tissue is the artefact perivascular space. Stained by haematoxylin and eosin. $\times 625$. 
attention was paid to the details of neuronal destruction or reaction.

In sections from the diseased brains and spinal cords the blood vessels appeared more conspicuous than in sections from normal material. This prominence may have been due largely to the well marked perivascular exudates which formed cuffs around the blood vessels. In sections stained by toluidine blue or by Mallory's trichrome stain the perivascular collections of cells can be clearly recognized (Figs. 4 and 5), but it is impossible to determine exactly their relationship to the reticular perivascular sheath. From the examination of these sections one cannot state unequivocally whether the cells are lying within the true perivascular space or in the artefact perivascular space, nor, indeed, whether they might not be lying within the adventitia (if this exists) of the blood vessel itself. On the other hand in sections which have been treated by the modification of Long's method the reticular perivascular sheath is readily distinguished (Fig. 6). In these latter sections it can be seen that the perivascular collections of inflammatory cells are, at least for the most part, contained within the Virchow-Robin spaces. They are separated from the wall of the blood vessel by

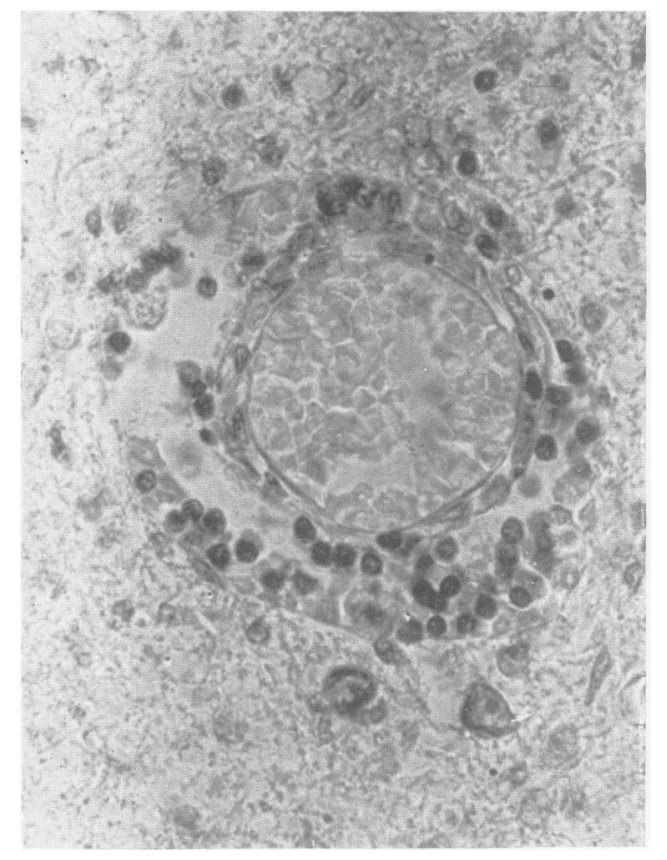

Fig. 4.-Transverse section through the spinal cord of a patient who died of poliomyelitis. The cellular exudate can be seen around the blood vessel (compare with Fig. 6). Stained with toluidine blue. $\times 500$. the inner layer of the reticular tissue and from the neurons by the outer lamina of the reticular sheath. Outside the reticular perivascular sheath can sometimes be seen a space, the artefact perivascular space, which is relatively free from cells (Fig. 7). Even quite small vessels show this perivascular cuffing and the separation of the reticular tissue into an inner and an outer layer, which in sections of normal tissue may not be seen, is now quite evident. The true perivascular spaces are closely packed with cells. It would appear that under pathological conditions the perivascular spaces are more extensive than in normal tissue. Whether this is due to the opening up of already existing potential spaces, which are not evident under normal conditions, or by the extension inwards of the perivascular spaces around the larger vessels splitting the reticular tissue into two layers, is difficult to determine. It would however seem unlikely that the latter process could occur to any marked extent.

In some of the sections stained with the modified Long's stain the outer lamina of perivascular reticular tissue shows a somewhat rough and shaggy appearance, in contrast to the sharply delineated appearance which it normally presents. The reticular

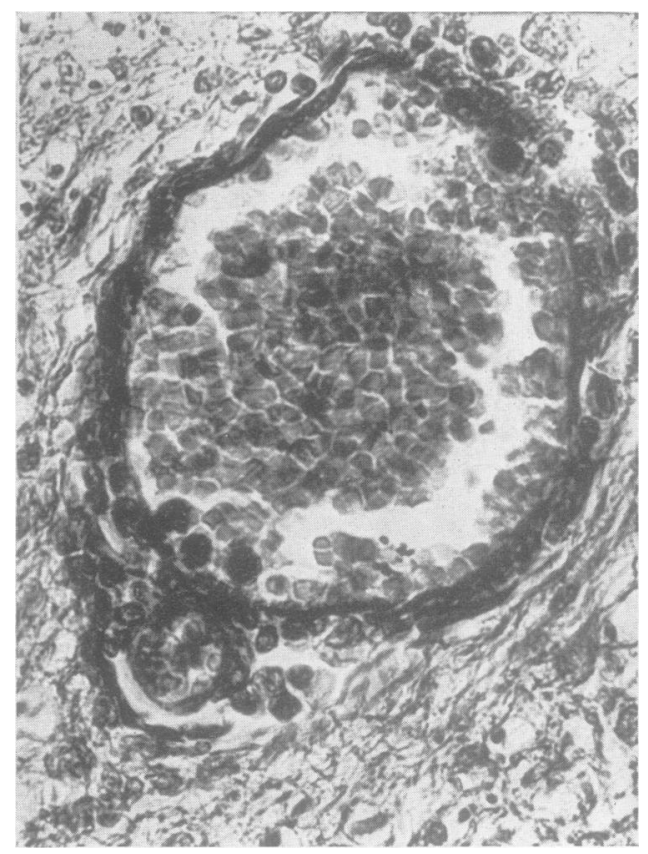

Fig. 5.-Transverse section through the same spinal cord as is shown in Fig. 4. The position of the cellular exudate in relation to the blood vessel is shown (compare with Fig. 6). Stained with Mallory's trichrome stain. $\times 500$. 


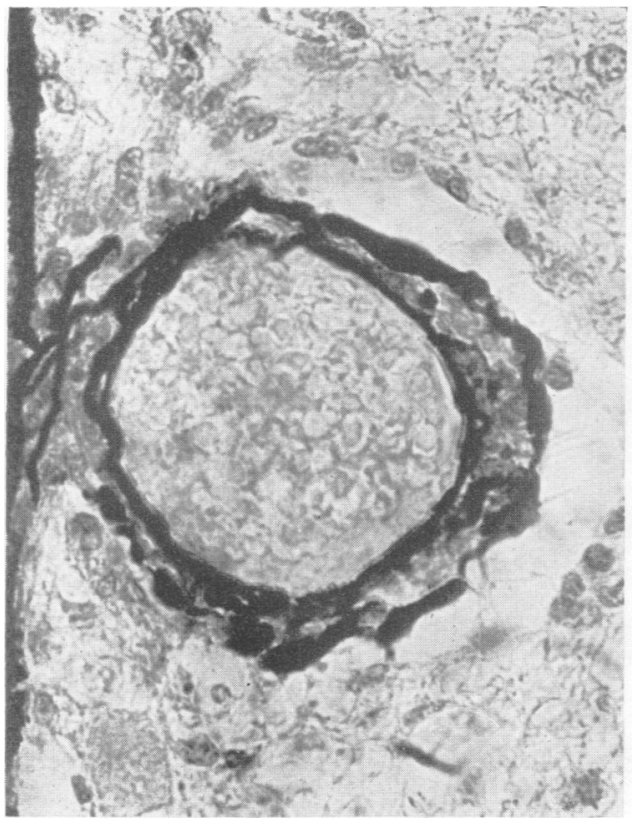

FIG. 6.-Transverse section through the same spinal cord as is shown in Fig. 4. The cellular exudate can clearly be seen to be confined to the space between the two layers of the reticular perivascular sheath, i.e., the true perivascular space. External to the outer layer of the sheath can be seen the artefact perivascular space. Stained by a modification of Long's stain. $\times 625$

tissue appears to be sending processes into the surrounding nervous tissue (Fig. 8). Such an appear-

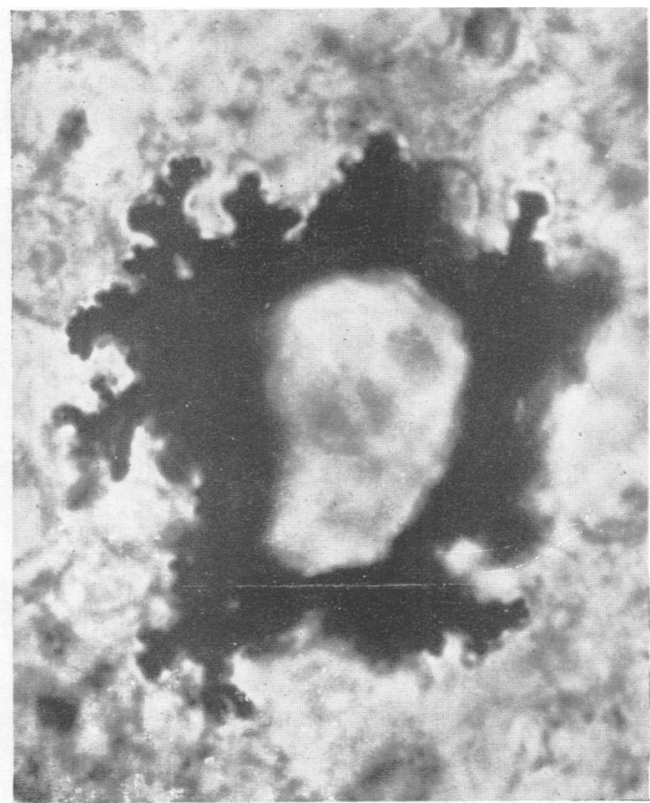

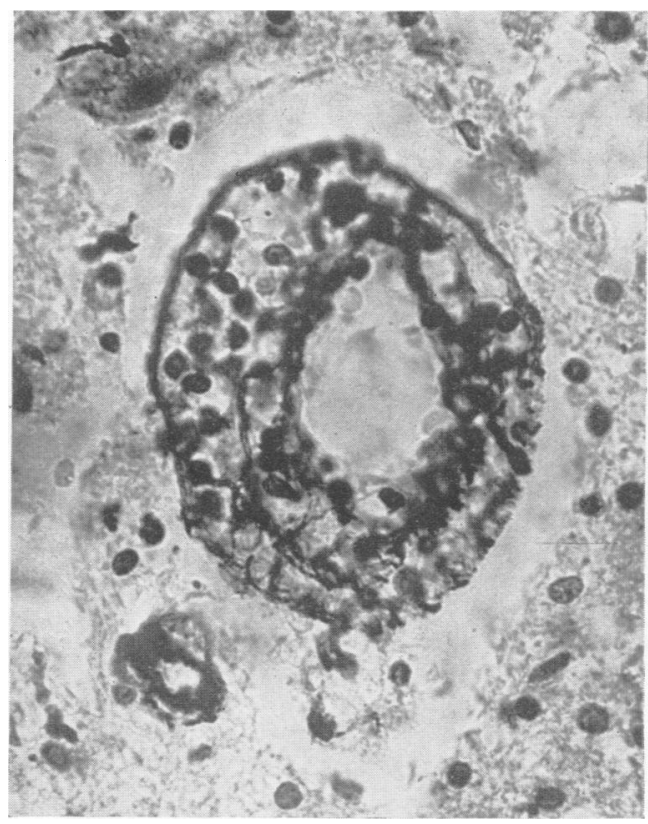

FIG. 7.-Transverse section through the spinal cord of a patient who died from encephalitis. The cellular exudate is seen to lie in the true perivascular space. External to the outer layer of the reticular perivascular sheath lies the artefact perivascular space, which contains only a few inflammatory cells. Stained by a modification of Long's stain. $\times 625$.

ance is highly suggestive of the occurrence of a proliferative reaction of the reticular tissue, although the material available for examination was insufficient to enable a definite decision to be reached on this point.

\section{Discussion}

Many of the misconceptions as to the nature, extent, and functions of the Virchow-Robin spaces have arisen from the failure to employ techniques which enabled the clear differentiation of the reticular perivascular sheaths to be made, and the recognition that this reticular tissue formed the inner and outer walls of the true perivascular spaces.

Before the evolution of silver and gold impregnation techniques workers were handicapped by their inability to distinguish clearly between the nervous and non-nervous elements of the central nervous system. It was, almost certainly, the absence of adequate staining methods that was responsible for the description by many of the earlier workers of the

FIG. 8.-Transverse section through the spinal cord of a patient who died from poliomyelitis. The proliferation into the surrounding nervous parenchyma of the reticular perivascular sheath of a small blood vessel may be seen. Stained by a modification of Long's stain. $\times 1820$. 
reticular perivascular sheath as the adventitia of the cerebral blood vessel (Virchow, 1851 ; Robin, 1859 ; His, 1865 ; Obersteiner, 1890 ; Bevan Lewis, 1889). There is little doubt that, for the most part, their accounts refer to the reticular perivascular sheath. Nevertheless the absence of an adequate staining method was almost certainly responsible for some unfortunate misconceptions that, up to the present day, have influenced views as to the functions of the perivascular spaces.

It is against this background that the classical work of Key and Retzius (1876) stands out as a landmark in the history of the reticular perivascular sheaths. They recognized that the blood vessels entering the brain were accompanied by endotheliallined perivascular spaces, and were the first to differentiate the reticular sheath into an outer layer derived from the pia mater and an inner derived from the arachnoid. They distinguished between the true perivascular space (of Virchow-Robin) and the artefact space described by His. In their account, however, they stated that the true perivascular space was intra-adventitial in position. It is evident that their use of the term adventitial was different from that commonly accepted to-day. It is unfortunate that this different usage of the term has contributed to the subsequent misinterpretation of their work.

Tuke (1894), while recognizing that the perivascular sheaths were derived from the leptomeninges and replaced the adventitial layer of the blood vessels within the central nervous system, believed that these sheaths surrounded the blood vessels to their ultimate branches and gave off processes which provided each neuron with a perineuronal capsule. At this distance in time it is impossible to say whether he was influenced in this belief by his conception of the perivascular system as responsible for the removal of waste and superfluous material from the nerve cells. The views of Mott (1910) have strongly influenced the beliefs of subsequent workers in this field : Cushing (1926), for example, was so impressed by Mott's work that he considered " no better description of the spaces existed in the English language". Mott's interest, like that of Tuke, arose primarily from his examinations of the brain in cases of insanity. Although Mott carried out some experimental work, his research was hampered by the absence of a suitable staining method for the reticular tissue and his failure to employ an indicator substance. His view as to the position of the perivascular space between the prolongations of the lepto -meninges is the generally accepted one but in his interpretation of sections of nervous tissue he wrongly identifies this space with the artefact space lying outside the reticular sheath. This error led him to expound the view that the perivascular spaces were part of a complex perivascular system continuous on the one hand with the perineuronal spaces and on the other with the subarachnoid space. A comparison of Mott's illustrations with sections stained to show the reticular tissue leaves no doubt that the spaces described by him as the true perivascular spaces are in fact the spaces observed by His (1865) who recognized them to be artefacts.

Although Weed (1914) did not employ histological techniques which would allow him to contribute to the better understanding of the reticular perivascular sheaths his contribution to the field of research into the circulation of the cerebrospinal fluid is such that his views cannot be ignored. Weed appears to have accepted without question the views expressed by Mott on the communication between the perineuronal and the perivascular spaces, thereby implying that there was no barrier interposed between the perivascular spaces and the nervous tissue by the outer layer of the reticular sheath. The experimental work of Weed, using the Prussian blue technique, was designed to answer problems concerned with the circulation in these channels of the cerebrospinal fluid and was not such as to enable him to verify or disprove the assumption upon which his work depended. The defects of the Prussian blue technique have been pointed out by Patek (1944) and their analysis is outside the scope of this article. It suffices to say that Weed's experiments did not throw any new light on the nature and arrangement of the reticular perivascular sheath.

Of more recent accounts the only ones that appear worthy of detailed consideration are those of Schaltenbrand and Bailey (1928) and Patek (1944). Schaltenbrand and Bailey (1928) based their descriptions essentially on the examination of pathological material. They were in agreement with Key and Retzius in recognizing the leptomeningeal nature of the perivascular sheaths. In one respect, however, they disagreed with earlier writers : they regarded the outer layer of the sheath as formed not only by the pia mater but also by the glia fused with it to form a " piaglialmembrane". On this concept of a "piaglialmembrane" was based their postulation of the to-and-fro movement of this membrane under certain conditions, thereby increasing or diminishing the size of the Virchow-Robin space at the expense of the artefact space. It may be noted that this view of the functioning of the perivascular spaces has not received the support of other workers in this field. Schaltenbrand and Bailey also considered that even the smallest capillaries 
were covered by a reticular perivascular sheath and surrounded by a Virchow-Robin space. Woollard (1924) was, however, of the opinion that the cell nuclei in relation to the capillaries, upon the presence of which they appear to have based their view, were neuroglial in origin. It is only fair to state that in a recent paper Schaltenbrand (1953) remarked that the perivascular spaces exist only round the larger vessels of the brain and that round the capillaries there is only a little connective tissue. If one ignores the thesis of a "piaglialmembrane" the views of Schaltenbrand and Bailey on the reticular perivascular sheaths accord very closely with those expressed earlier by Key and Retzius (1876).

The work of Patek (1944) on the reticular sheaths and the perivascular spaces does not appear to have received the recognition which it merited. Patek employed colloidal mercuric sulphide as an indicater and combined its use with that of intravenous hypertonic saline. From his experiments and subsequent histological examinations he concluded that the inner layer of the reticular perivascular sheath was derived from the arachnoid mater. He considered that this layer accompanied the arteries and veins as far as the arterioles and venules as a complete membrane. Patek was unable to recognize a continuous covering over the capillaries. His view of the outer layer of the perivascular sheath was unique in that he regarded the pia mater as extending along the arteries for only a very short distance and being then replaced by a fenestrated membrane. On the veins the pia mater extended as far as the venules and then terminated in the same manner. In the opinion of Patek the outer wall of the perivascular space was formed, in its deeper part, by a glial membrane. Patek's work implies that the inner and outer walls of the reticular sheaths do not fuse at the inner ends of the perivascular spaces and indeed he describes the fluid in the perivascular spaces as becoming continuous with the tissue fluid of the brain. The views of Patek with regard to the termination of the outer wall of the perivascular space are not borne out by the present investigation. In sections stained by the modified Long's method it can be seen clearly that the outer wall of the perivascular space formed by reticular tissue continuous with the pia mater on the surface of the nervous system extends much deeper into the nervous tissue than he suggests. Furthermore, if the reticular sheaths are open at their inner ends it is difficult to see the mechanism by which the flask-shaped distension of the perivascular spaces is produced in torula meningitis (Schaltenbrand and Bailey, 1928).

When one considers the confusion that exists concerning the normal histology of the reticular perivascular tissue it is not perhaps surprising to find that the relationship of these reticular perivascular sheaths to pathological processes is subject to the same confusions and misconceptions. Even in standard textbooks (Boyd, 1950) one finds the Virchow-Robin spaces described as adventitial spaces whilst the artefact perivascular spaces (of His) are regarded as the perivascular spaces proper. The use of a suitable reticular stain makes it possible to identify clearly the inner and outer layers of the reticular sheath, and to recognize that the inflammatory cells around the vessels are essentially confined to the true perivascular spaces (of VirchowRobin) bounded by these layers. The perivascular spaces often appear to be completely filled, and perhaps even distended, by densely packed inflammatory cells. This finding, together with the observation that in some instances the reticular tissue appears to be undergoing a proliferative reaction, suggests that the reticular perivascular sheaths may play a less passive role in the processes of disease than is generally attributed to them.

\section{Summary}

The clear identification of the reticular perivascular tissue of the central nervous system is important to the better understanding of the relationships between this tissue and the blood vessels on the one hand and the nervous tissue on the other.

Much of the confusion in the literature concerning the reticular perivascular tissue derived from the failure of investigators to employ suitable histological techniques for its definition. In consequence the artefact perivascular space has often been identified wrongly with the true perivascular (Virchow-Robin) space.

A modification of Long's (1948) stain for reticular and collagenous fibrils was applied to the study of material from the central nervous systems of laboratory rats. These rats had received daily subarachnoid injections of colloidal carbon for a period of three weeks from birth before being sacrificed. Sections from the brains and spinal cords of patients who had died following attacks of poliomyelitis and epidemic encephalitis were also stained by this method.

The examination of the normal material confirmed the continuity of the outer layer of the reticular perivascular sheath with the pia mater and of the inner layer with the arachnoid mater. It was found that the reticular tissue surrounded all the blood vessels within the substance of the central nervous system with the possible exception of the smaller capillaries.

The reticular stain rendered easy the recognition and delimitation of the perivascular (Virchow- 
Robin) spaces and made it possible to distinguish unequivocally these spaces from the artefact perivascular spaces.

The application of the reticular stain to pathological material enabled the position of perivascular exudates within the true perivascular spaces to be clearly recognized. The examination of the sections revealed also a shaggy appearance of the reticular perivascular tissue which suggested that this tissue might perhaps be undergoing a proliferative reaction in response to the processes of disease.

We wish to thank Professor J. D. Boyd for his advice and encouragement. Our thanks are also due to Professor Dorothy Russell and Dr. A. M. Barrett for the supply of material, to Mr. T. R. L. Brooks for photography, and to Mr. R. Smith for technical assistance.

\section{REFERENCES}

Boyd, W. (1950). The Pathology of Internal Diseases, 5th ed., p. 721. Kimpton, London.

Bruce, A., and Dawson, J. W. (1911). J. Path. Bact., 15, 169.

Cushing, H. (1926). Studies in Intracranial Physiology and Surgery. The Third Circulation, The Hypophysis, The Gliomas, p. 16. Oxford University Press, London.

His, W. (1865). Z. wiss. Zool., 15, 127.

Hughson, W. (1925). Arch. Neurol. Psychiat., Chicago, 14, 240.

Key, A., and Retzius, G. (1876). Studien in der Anatomie des Nervensystems und des Bindegewebes. Samson and Wallin, Stockholm.

Lewis, W. Bevan. (1889). A Text-book of Mental Diseases; with Special Reference to the Pathological Aspects of Insanity, p. 81. Griffin, London.

Long, M. E. (1948). Stain Tech., 23, 69.

Mott, F. W. (1910). Lancet, 2 , i.

Obersteiner, H. (1890). The Anatomy of the Central Nervous Organs in Health and in Disease, $\mathrm{p}, 138$. Griffin, London.

Patek, P. R. (1944). Anat. Rec., 88, 1.

Robin, C. (1859). J. Physiol., Paris., ?, 537.

Schaltenbrand, G. (1953). Lancet, 1, 805.

-, and Bailey, P. (1928). J. Psychol. Neurol., Lpz., 35, 199.

Tuke, J. B. (1894). Edinb. med. J., 39, 673.

Virchow, R. (1851). Virchow's Arch. path. Anat., 3, 427.

Weed, L. H. (1914). J. med. Res., 31, 21.

Woollard, H. H. (1924). J. Anat., Lond., 58, 89. 